\title{
Concordance of genetic variation that increases risk for Tourette Syndrome and that influences its underlying neurocircuitry
}

Mary Mufford (1) ', Josh Cheung ${ }^{2}$, Neda Jahanshad², Celia van der Merwe', Linda Ding², Nynke Groenewold ${ }^{3}$, Nastassja Koen ${ }^{3,4}$, Emile R. Chimusa (1) ', Shareefa Dalvie ${ }^{3,4}$, Raj Ramesar ${ }^{1}$, James A. Knowles ${ }^{5}$, Christine Lochner ${ }^{6}$, Derrek P. Hibar ${ }^{2}$, Peristera Paschou', Odile A. van den Heuvel ${ }^{8}$, Sarah E. Medland ${ }^{9}$, Jeremiah M. Scharf ${ }^{10,11,12}$, Carol A. Mathews ${ }^{13}$, Paul M. Thompson ${ }^{2}$ and Dan J. Stein ${ }^{3,4}$, Psychiatric Genomics Consortium - Tourette Syndrome working group

\begin{abstract}
There have been considerable recent advances in understanding the genetic architecture of Tourette Syndrome (TS) as well as its underlying neurocircuitry. However, the mechanisms by which genetic variation that increases risk for TSand its main symptom dimensions-influence relevant brain regions are poorly understood. Here we undertook a genome-wide investigation of the overlap between TS genetic risk and genetic influences on the volume of specific subcortical brain structures that have been implicated in TS. We obtained summary statistics for the most recent TS genome-wide association study (GWAS) from the TS Psychiatric Genomics Consortium Working Group (4644 cases and 8695 controls) and GWAS of subcortical volumes from the ENIGMA consortium (30,717 individuals). We also undertook analyses using GWAS summary statistics of key symptom factors in TS, namely social disinhibition and symmetry behaviour. SNP effect concordance analysis (SECA) was used to examine genetic pleiotropy-the same SNP affecting two traits - and concordance - the agreement in single nucelotide polymorphism (SNP) effect directions across these two traits. In addition, a conditional false discovery rate (FDR) analysis was performed, conditioning the TS risk variants on each of the seven subcortical and the intracranial brain volume GWAS. Linkage disequilibrium score regression (LDSR) was used as validation of the SECA method. SECA revealed significant pleiotropy between TS and putamen ( $p=$ $\left.2 \times 10^{-4}\right)$ and caudate $\left(p=4 \times 10^{-4}\right)$ volumes, independent of direction of effect, and significant concordance between TS and lower thalamic volume $\left(p=1 \times 10^{-3}\right)$. LDSR lent additional support for the association between TS and thalamus volume $\left(p=5.85 \times 10^{-2}\right)$. Furthermore, SECA revealed significant evidence of concordance between the social disinhibition symptom dimension and lower thalamus volume $\left(p=1 \times 10^{-3}\right)$, as well as concordance between symmetry behaviour and greater putamen volume $\left(p=7 \times 10^{-4}\right)$. Conditional FDR analysis further revealed novel variants significantly associated with TS $\left(p<8 \times 10^{-7}\right)$ when conditioning on intracranial (rs2708146, $q=0.046$; and rs72853320, $q=0.035$ ) and hippocampal (rs1922786, $q=0.001$ ) volumes, respectively. These data indicate concordance for genetic variation involved in disorder risk and subcortical brain volumes in TS. Further work with larger samples is needed to fully delineate the genetic architecture of these disorders and their underlying neurocircuitry.
\end{abstract}

\footnotetext{
Correspondence: Dan J. Stein (dan.stein@uct.ac.za)

${ }^{1}$ Human Genetics Research Unit, Division of Human Genetics, Department of Pathology, Institute of Infectious Disease and Molecular Medicine, Faculty of Health Sciences, University of Cape Town, Cape Town, South Africa

${ }^{2}$ Imaging Genetics Center, Mark and Mary Stevens Neuroimaging \& Informatics Institute, Keck School of Medicine of the University of Southern California, Los Angeles, CA, USA
}

Full list of author information is available at the end of the article.

\section{Introduction}

Tourette's Syndrome (TS) has a global prevalence of $\sim 0.85-1 \%^{1}$ and is characterised by repetitive motor and phonic tics, with onset typically before the age of 18 years $^{2}$. TS has one of the highest heritability estimates for

\section{(c) The Author(s) 2019}

\footnotetext{
(c) Open Access This article is licensed under a Creative Commons Attribution 4.0 International License, which permits use, sharing, adaptation, distribution and reproduction c. in any medium or format, as long as you give appropriate credit to the original author(s) and the source, provide a link to the Creative Commons license, and indicate if changes were made. The images or other third party material in this article are included in the article's Creative Commons license, unless indicated otherwise in a credit line to the material. If material is not included in the article's Creative Commons license and your intended use is not permitted by statutory regulation or exceeds the permitted use, you will need to obtain permission directly from the copyright holder. To view a copy of this license, visit http://creativecommons.org/licenses/by/4.0/.
} 
neuropsychiatric disorders $(70-80 \%)^{3}$, with $50-60 \%$ of this heritability directly attributable to single nucleotide polymorphisms (SNPs) ${ }^{4}$. In recent years, there have been significant advances in understanding the genetic architecture of TS and in delineating other aspects of its underlying neurobiology, including its specific neuroanatomy ${ }^{1}$.

The Psychiatric Genomics Consortium Tourette Syndrome working group (PGC-TS) undertook the first genome-wide association study (GWAS) of TS, comprising 1285 cases and 4964 controls $^{5}$. While no SNP reached genome-wide significance, the top-ranking variants were enriched for genes that affect gene expression and methylation levels in the fronto-striatal circuitry, consistent with contemporary models of $\mathrm{TS}^{6}$. The lack of genome-wide significance at this sample size likely reflects the polygenic and heterogeneous nature of $\mathrm{TS}^{7-9}$, which is further complicated by comorbidity with other psychiatric disorders, such as obsessive-compulsive disorder (OCD), autism spectrum disorders (ASD) and attention-deficit/ hyperactivity disorder (ADHD $)^{10}$. Another study used the most highly associated variants from the PGC-TS GWAS to predict TS status $(p=0.042)$ in an independent cohort (609 cases and 610 controls) and they accounted for $0.52 \%$ of the variance observed between cases and controls ${ }^{11}$.

Several studies have attempted to clarify the complex nature of TS by identifying more homogenous endophenotypes and symptom dimensions ${ }^{12-15}$. While these studies identified several classes of Tourette-related endophenotypes using multivariate methods, all were based on relatively small samples $(n<1000)^{13-15}$. A recent and considerably larger analysis of individuals with TS and their family members that assessed not only TS, but also OCD and ADHD $\left(n_{\text {total }}=3494\right)$, identified two crossdisorder symptom dimensions, namely social disinhibition and symmetry behaviour ${ }^{16}$. Social disinhibition includes uttering syllables/words, echolalia/palilalia, coprolalia/copropraxia, and obsessive urges to offend/ mutilate/be destructive. Symmetry behaviour includes symmetry, evening up, checking obsessions, ordering, arranging, counting, writing-rewriting compulsions and repetitive writing tics. Social disinhibition $\left(h^{2}=0.35 \pm\right.$ 0.03 ) was associated with OCD polygenic risk scores (PRS; $p=0.02)$ and less strongly with TS and ADHD PRS, which did not meet statistical significance $(p=0.11$ and $p=0.10$, respectively). In contrast, symmetry behaviour $\left(h^{2}=0.39 \pm 0.03\right)$ was significantly correlated with TS PRS $(p=0.02)$ and not with OCD and ADHD $(p=0.18$ and $p=0.26$, respectively).

There have also been noteworthy advances in the understanding of the neuronal circuitry of TS. The role of the cortico-striatal-thalamo-cortical circuits (CSTC) in TS has been emphasized ${ }^{17}$, although data on changes in the volume and function of specific brain regions in the
CSTC in individuals with TS is less consistent. Lower bilateral nucleus caudate volumes ${ }^{18,19}$, inferior occipital volumes $^{20}$, prefrontal cortex volumes ${ }^{20,21}$, corpus callosum volumes and decreased white matter connectivity ${ }^{22,23}$ have been observed in children with TS. Greater grey matter volumes have also been observed in the thalamus $^{21,24}$, hypothalamus and midbrain among children and adults with $\mathrm{TS}^{21,25}$. Amygdalar volume has been reported to be greater in children and lower in adulthood $^{25}$.

Little work to date has, however, focused on pleiotropy or concordance of genetic risk for TS, and genetic variants that influence subcortical brain volume. Pleiotropy refers to a SNP that affects both phenotypes, regardless of whether the effect direction is the same for both. Concordance, however, requires that the SNP has the same direction of effect for both phenotypes. The Enhancing Neuroimaging Genetics through Meta-analysis (ENIGMA) consortium recently performed a GWAS of structural brain MRI scans of 30,717 individuals ${ }^{26}$. The ENIGMA subcortical brain volumes study identified novel genetic variants associated with the volumes of the putamen and caudate nucleus ${ }^{26}$ and subsequently detected an overlap with OCD risk variants ${ }^{27}$. ENIGMA provides an opportunity to examine the relationship between GWAS data in TS with the genetic contributions to regional brain volumes. Here we aim to assess genetic concordance for TS and specific symptom profiles (i.e., social disinhibition and symmetry behaviour) with the volume of relevant subcortical and intracranial brain regions. We used summary statistics from the ENIGMA subcortical and intracranial brain volumes GWAS $^{26}$ and the most recent PGC-TS GWAS ${ }^{5}$.

\section{Methods \\ Description of original association studies}

We obtained summary statistics of adult European ancestry participants (4644 cases and 8695 controls) and 9,076,550 SNPs from the most recent PGC-TS GWAS ${ }^{5}$, including unpublished data. Approximately half of this cohort also had either comorbid OCD or ADHD. A subset of cases had information regarding symmetry behaviour $(n=1419)$ and social disinhibition $(n=1414)$ symptom classes. Participants were diagnosed using the DSM-IV$\mathrm{TR}^{28}$ by trained clinicians. In addition, we used GWAS summary statistics from the ENIGMA Consortium metaanalysis of subcortical brain volumes across 50 cohorts, including MRI scans of 30,717 individuals and 9,702,043 $\mathrm{SNPs}^{26}$. This cohort consisted of healthy controls (79\%) as well as patients $(21 \%)$ diagnosed with neuropsychiatric disorders (including anxiety disorders, Alzheimer's disease, ADHD, major depression, bipolar disorder, epilepsy and schizophrenia). A direct comparison of the GWAS summary statistics between the full ENIGMA results 
(including patients) and a subset of ENIGMA results (excluding patients) showed that they were very highly correlated (Pearson's $r>0.99$ ) for all brain traits ${ }^{26}$. Prior to the analyses here, we verified that there was no cohort overlap between the TS and brain volume GWASs and therefore individual overlap was likely to be minimal, if any at all. The brain volume GWAS data comprised GWASs of seven subcortical brain volumes (nucleus accumbens, amygdala, caudate nucleus, hippocampus, globus pallidus, putamen, thalamus) and total intracranial volume (ICV). GWAS test statistics were genomecontrolled to adjust for spurious inflation factors. All cohort studies were approved by a local ethics board prior to subject recruitment and all subjects gave written, informed consent before participating.

\section{Post-processing of genetic data}

To statistically compare the TS and brain volume GWASs, we used the 7,682,991 SNPs that passed quality control and filtering rules in all datasets. With these data, we performed a clumping procedure in $\mathrm{PLINK}^{29}$ to identify an independent SNP from every linkage disequilibrium (LD) block across the genome. The clumping procedure was performed separately for each of the eight brain volume GWASs using a $500 \mathrm{~kb}$ window, with SNPs in $\operatorname{LD}\left(r^{2}>0.2\right)$, in the European reference samples from the 1000 Genome Project (Phase 1, version 3). The SNP with the lowest $p$-value within each LD block was selected as the index SNP representing that LD block and all other SNPs in the LD block were dropped from the analysis. The result, after applying the clumping procedure, was a total of eight independent sets of SNPs representing the total variation explained across the genome, conditioned on the significance in each brain volume GWAS. For each of these eight sets of SNPs, we then determined the corresponding TS GWAS test statistic for each independent index SNP and used these datasets for the subsequent analyses.

\section{Tests of pleiotropy and concordance}

We used SNP effect concordance analysis (SECA) ${ }^{30}$ (see Nyholt 2014 for details of the SECA analysis) to test for genetic pleiotropy-the same SNP affecting two traitsand concordance-the agreement in SNP effect directions across these two traits-between TS, social disinhibition or symmetry behaviour and all seven subcortical structures and ICV.

\section{Conditional false discovery rate to detect TS, social disinhibition or symmetry behaviour risk variants}

We also examined if conditioning the results of the TS GWAS on genetic variants that influence subcortical brain volume (TS | subcortical brain volume) could improve our ability to detect variants associated with
$\mathrm{TS}^{31,32}$. At this sample size, the analyses were underpowered to investigate the symptom dimensions separately. For a given brain volume phenotype, we selected subsets of SNPs at 14 false discovery rate (FDR) thresholds ( $q$-values $\leq 1 \times 10^{-5}, 1 \times 10^{-4}, 1 \times 10^{-3}, 0.01,0.1,0.2$, $0.3,0.4,0.5,0.6,0.7,0.8,0.9,1)$ and looked up the corresponding $p$-values for each SNP subset in the TS GWAS and the social disinhibition and symmetry behaviour symptom clusters. Next, we applied the FDR method ${ }^{33}$ to each subset of $p$-values in the TS GWAS and the social disinhibition and symmetry behaviour symptom clusters. Individual SNPs were considered significant if the $p$-value was lower than the significance threshold, allowing for an FDR of 5\%, conditioned on any subset of SNPs from the brain volume GWASs. The LD-pruned data were required for the conditional FDR SNP analysis because regions with varying amounts of SNPs within an LD block can affect the ranking and re-ranking of SNPs under the conditional models. However, the chosen SNP included in the model is likely just a "proxy" for SNPs in the LD block and should not necessarily be considered a causal variant or even the most significant SNP in terms of its overlap between traits.

Stratified true discovery rate (TDR) plots were constructed by subsetting SNPs based on associations with a secondary trait (i.e. subcortical brain volumes), and generating TDR plots separately for each subset of SNPs based on their association with the main trait of interest (i.e. TS). The SNPs that had reached at least marginal significance thresholds for the relevant subcortical brain regions were selected for inclusion in the plots. The selected $p$-value thresholds were 1.0, 0.1, 0.01 and 0.001 , which were log-transformed. A conservative measure of TDR is calculated for each SNP as $1-(p / q)$, where $p$ represents the $p$-value of a SNP's association with the primary trait of interest (TS), and $q$ represents the empirical conditional cumulative distribution function $q$ (TS | subcortical brain region) $)^{34}$.

\section{Estimating genetic correlation using LD score regression}

In order to replicate significant findings from our primary analysis with SECA, we used an alternative method, LD score regression (LDSR), which estimates a genetic correlation between two trait pairs based on the GWAS summary statistics of each trait analysed separately ${ }^{35,36}$. LDSR estimates a genetic correlation with a fitted linear model of Z-scores obtained from the product of significance statistics for each SNP in a given set of GWAS results compared to the level of linkage disequilibrium at a given SNP. SNPs in high LD are expected to have high Z-scores in polygenic traits with common genetic over$\mathrm{lap}^{35}$. We used the ldsc program (https://github.com/ bulik/ldsc) to perform LDSR following the methods outlined in Bulik-Sullivan et al., 2015 ${ }^{36}$. LDSR was 
underpowered at this sample size to analyse the amygdala, as well as the TS symptom clusters. Therefore, LDSR was only used to test for an association with the broader TS phenotype and the remaining seven brain regions of interest. Given the number of tests performed, we set a Bonferroni corrected significance level at $p^{*}=0.05 / 48=$ $1.042 \times 10^{-3}$ to account for the three traits tested (TS, social disinhibition and symmetry behaviour), the eight brain volumes and the two tests performed (SECA and LDSR).

\section{Results}

Evidence for pleiotropy between brain volume and TS and related symptom cluster risk variants

We found significant evidence of global pleiotropysame SNP, regardless of effect direction-between variants that infer risk for the broader TS phenotype and variants that are associated with lower putamen (Table 1 , $p=2 \times 10^{-4}$ ) and caudate volumes (Table $1, p=4 \times$ $\left.10^{-4}\right)$. Further, we found nominally significant $(p<0.05)$ evidence of pleiotropy between TS risk variants and variants associated with lower ICV, accumbens, pallidum, and thalamus volumes and greater hippocampus volume (Table 1). No evidence for global pleiotropy was found for either the social disinhibition (Table 2) or symmetry behaviour (Table 3) symptom clusters.

Evidence for concordance between brain volumes and TS and related symptom clusters risk variants

We found significant evidence of negative concordancesame SNP, same direction of effect-between TS and thalamus volume (Table $1, p=3 \times 10^{-4}$ ), indicating an association between TS genetic risk and lower thalamus volume. Further, we found nominally significant $(p<0.05)$ negative concordance between ICV and caudate volumes with TS (Table 1). Nominally significant positive concordance was found between the amygdala and TS. Significant negative concordance was also identified between the social disinhibition behaviour symptom cluster and the thalamus (Table $2, p=1 \times 10^{-3}$ ) as well as marginally significant negative concordance with the accumbens $\left(p=2.3 \times 10^{-3}\right)$ and positive concordance with the pallidum $\left(p=0.47 \times 10^{-2}\right)$. Significant positive concordance was also identified between putamen volume and symmetry behaviour (Table $3, p=7 \times 10^{-4}$ ). Evidence for marginally significant negative concordance between this symptom cluster and the amygdala $\left(p=2 \times 10^{-3}\right)$ and positive concordance with the caudate $\left(p=1.7 \times 10^{-2}\right)$ were also observed.

\section{Replication of subcortical brain volumes and TS genetic risk overlap using LDSR}

Replication using LDSR lent trending support for an association between TS risk and the thalamus (Table 4, $p=5.85 \times 10^{-2}$ ), although this was not significant. No association between TS and pallidum volume was observed.

\section{Genetic variants influencing subcortical brain volumes provide improved ability to detect TS risk variants}

We performed a conditional FDR analysis, conditioning the TS risk variants on each of the eight brain volume GWASs (Table 5). When conditioning the TS analysis on variants that influence ICV, rs2708146 $(q=0.046)$ and rs72853320 $(q=0.035)$ were significantly associated with both traits. Conditioning TS on the hippocampus also revealed an association between $\operatorname{rs} 1922786(q=0.001)$. Each of these variants account for $<1 \%$ of the variance observed in TS, as well as in each of the associated brain region volumes. No significant associations $(q<0.05)$ were identified when conditioning TS on the other six brain GWAS.

\section{Table 1 SECA results for TS whole cohort}

\begin{tabular}{|c|c|c|c|c|c|c|}
\hline Trait 1 & Trait 2 & $p$-value pleiotropy & Cl pleiotropy & $p$-value concordance & $\mathrm{Cl}$ concordance & Direction \\
\hline \multirow[t]{8}{*}{ TS } & Intracranial volume & $0.001^{* *}$ & $0.001-0.002$ & $0.022^{* *}$ & $0.019-0.025$ & - \\
\hline & Accumbens & $0.007^{* *}$ & $0.006-0.009$ & $0.054^{*}$ & $0.049-0.058$ & - \\
\hline & Amygdala & 1 & $1-1$ & $0.048^{* *}$ & $0.044-0.053$ & + \\
\hline & Caudate & $4 \times 10^{-4 * * *}$ & $1 \times 10^{-4}-0.001$ & $0.016^{* *}$ & $0.013-0.018$ & - \\
\hline & Hippocampus & $0.009 * *$ & $0.007-0.011$ & $0.07^{*}$ & $0.065-0.075$ & + \\
\hline & Pallidum & $0.006^{* *}$ & $0.004-0.007$ & 0.257 & $0.249-0.266$ & - \\
\hline & Putamen & $2 \times 10^{-4 * * *}$ & $5.48 \times 10^{-5}-0.007$ & 1 & $1-1$ & - \\
\hline & Thalamus & $0.009^{* *}$ & $0.008-0.012$ & $3 \times 10^{-4 * * *}$ & $1 \times 10^{-4}-0.001$ & - \\
\hline
\end{tabular}

TS Tourette's syndrome, $\mathrm{Cl}$ confidence interval, Bonferroni corrected $p$-value $=0.05 / 48=1.042 \times 10^{-3}$

*Trending significance $(p<0.1)$

**Nominally significant $(p<0.05)$

***Significant 
Table 2 SECA results for TS social disinhibition endophenotype

\begin{tabular}{|c|c|c|c|c|c|c|}
\hline Trait 1 & Trait 2 & $p$-value pleiotropy & $\mathrm{Cl}$ pleiotropy & $p$-value concordance & $\mathrm{Cl}$ concordance & Direction \\
\hline \multirow[t]{8}{*}{ TS } & Intracranial volume & 1 & $1-1$ & 1 & $1-1$ & + \\
\hline & Accumbens & 0.31 & $0.301-0.319$ & $0.002^{* *}$ & $0.002-0.003$ & - \\
\hline & Amygdala & 0.166 & $0.159-0.173$ & $0.084^{*}$ & $0.079-0.09$ & - \\
\hline & Caudate & 1 & $1-1$ & 0.166 & $0.159-0.173$ & - \\
\hline & Hippocampus & 1 & $1-1$ & $0.099^{*}$ & $0.093-0.105$ & - \\
\hline & Pallidum & 1 & $1-1$ & $0.047^{* *}$ & $0.043-0.051$ & + \\
\hline & Putamen & 1 & $1-1$ & 0.194 & $0.186-0.201$ & - \\
\hline & Thalamus & 1 & $1-1$ & $1 \times 10^{-3 * * *}$ & $5.13 e-06-0.001$ & - \\
\hline
\end{tabular}

TS Tourette's syndrome, $\mathrm{Cl}$ confidence interval, Bonferroni corrected $p$-value $=0.05 / 48=1.042 \times 10^{-3}$

*Trending significance $(p<0.1)$

${ }^{* *}$ Nominally significant $(p<0.05)$

***Significant

Table 3 SECA results for TS symmetry endophenotype

\begin{tabular}{|c|c|c|c|c|c|c|}
\hline Trait 1 & Trait 2 & $p$-value pleiotropy & $\mathrm{Cl}$ pleiotropy & $p$-value concordance & $\mathrm{Cl}$ concordance & Direction \\
\hline \multirow[t]{8}{*}{ TS } & Intracranial volume & 1 & $1-1$ & 0.191 & $0.183-0.198$ & - \\
\hline & Accumbens & 0.304 & $0.296-0.314$ & 0.263 & $0.255-0.272$ & - \\
\hline & Amygdala & 0.298 & $0.289-0.307$ & $0.002^{* *}$ & $0.001-0.003$ & - \\
\hline & Caudate & 0.304 & $0.295-0.313$ & $0.017^{* *}$ & $0.015-0.02$ & + \\
\hline & Hippocampus & 1 & $1-1$ & $0.07^{*}$ & $0.065-0.075$ & + \\
\hline & Pallidum & 1 & $1-1$ & 0.417 & $0.408-0.427$ & + \\
\hline & Putamen & 0.313 & $0.304-0.322$ & $0.001^{* * *}$ & $0-0.001$ & + \\
\hline & Thalamus & 1 & $1-1$ & 1 & $1-1$ & - \\
\hline
\end{tabular}

TS Tourette's syndrome, $\mathrm{Cl}$ confidence interval, Bonferroni corrected $p$-value $=0.05 / 48=1.042 \times 10^{-3}$

*Trending significance $(p<0.1)$

${ }^{* *}$ Nominally significant $(p<0.05)$

***Significant

The conditional TDR reflects the posterior probability that a given SNP is truly associated with the first phenotype (i.e. TS) given that the $p$-values for both phenotypes are as small or smaller than the observed $p$-values. The TDR plots show an increase in TDR associated with increased pleiotropic enrichment in TS conditional on nominal $p$-values for (i) ICV (Fig. 1) and (ii) hippocampus volume (Fig. 2). The successive leftward shifts for decreasing nominal $p$-value thresholds of both ICV and hippocampus volumes indicate that the proportion of SNPs with non-null effects on TS varies considerably across various levels of association with each of these subcortical brain volumes.

\section{Discussion}

Of the eight brain traits investigated using SECA, associations were found between genetic risk for TS and for lower thalamus, putamen and caudate volumes. These
Table 4 LDSR results for TS whole cohort and brain volume overlap

\begin{tabular}{llllll}
\hline Trait 1 & Trait 2 & rg & SE & 95\% Cl & p-value \\
\hline TS & Intracranial volume & -0.122 & 0.075 & $-0.269-0.025$ & 0.106 \\
& Accumbens & -0.428 & 0.582 & $-1.568-0.712$ & 0.462 \\
Amygdala & - & - & - & - \\
Caudate & -0.037 & 0.087 & $-0.208-0.134$ & 0.672 \\
Hippocampus & -0.015 & 0.097 & $-0.205-0.175$ & 0.878 \\
& Pallidum & -0.002 & 0.106 & $-0.21-0.206$ & 0.983 \\
Putamen & -0.023 & 0.091 & $-0.201-0.155$ & 0.801 \\
Thalamus & -0.227 & 0.12 & $-0.462-0.008$ & $0.059^{*}$ \\
\hline
\end{tabular}

TS Tourette's syndrome, $\mathrm{Cl}$ confidence interval, Bonferroni corrected $p$-value $=$ $0.05 / 48=1.042 \times 10^{-3}$

*Trending significance $(p<0.1)$ 

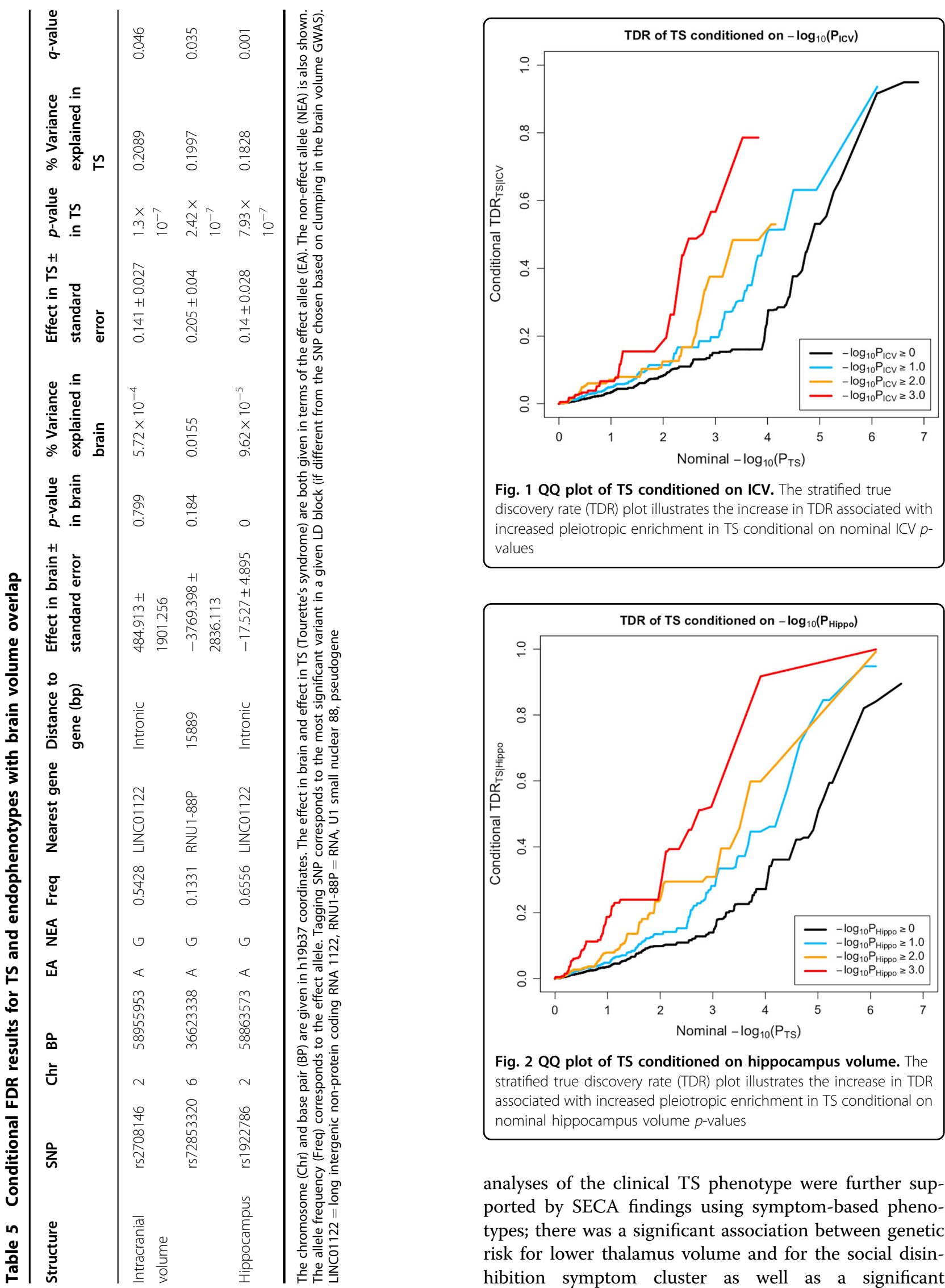

Fig. 1 QQ plot of TS conditioned on ICV. The stratified true discovery rate (TDR) plot illustrates the increase in TDR associated with increased pleiotropic enrichment in TS conditional on nominal ICV pvalues

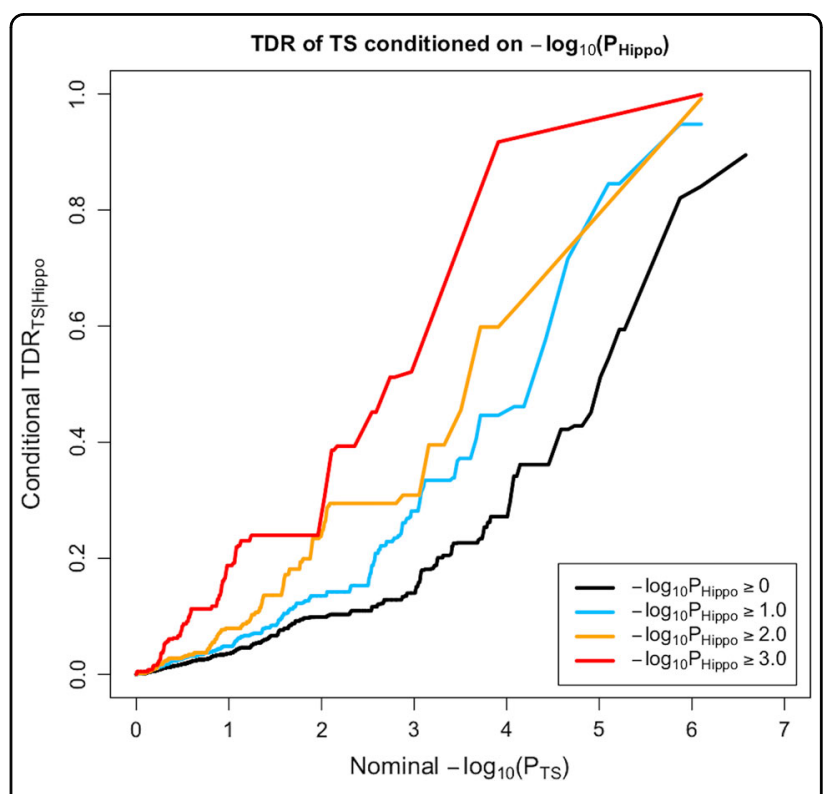

Fig. 2 QQ plot of TS conditioned on hippocampus volume. The stratified true discovery rate (TDR) plot illustrates the increase in TDR associated with increased pleiotropic enrichment in TS conditional on nominal hippocampus volume $p$-values

analyses of the clinical TS phenotype were further supported by SECA findings using symptom-based phenotypes; there was a significant association between genetic risk for lower thalamus volume and for the social disinhibition symptom cluster as well as a significant 
association between genetic risk for greater putamen volume and for the symmetry symptom cluster. Further, three SNPs were associated with both TS and the volumes of the hippocampus and ICV.

Associations of genetic risk for TS with genetic risk for alterations in thalamus and striatum volumes is consistent with the emphasis of neuroanatomical models of TS on these structures ${ }^{37,38}$. The thalamus has widespread projections affecting many aspects of cognition and motor function $^{39}$, and is a target for deep brain stimulation in the treatment of refractory $\mathrm{TS}^{40}$. While greater thalamus volumes have been reported in $\mathrm{TS}^{21,24}$, lower thalamus volumes have been identified in paediatric patients with $\mathrm{TS}^{41}$. The dorsal striatum is also involved in motor function $^{42}$, as well as various types of learning ${ }^{43,44}$, inhibitory control of action, and reward systems ${ }^{45}$. Evidence for both greater $^{46,47}$ and lower putamen volumes ${ }^{18,21,48}$ has been reported in both children and adults with TS, with lower putamen volumes reported in TS with comorbid OCD ${ }^{18}$. Lower caudate volumes in adults and children have been associated with $\mathrm{TS}^{18,48}$, particularly apparent in individuals with comorbid $\mathrm{ADHD}^{18}$, and are associated with tic severity and OCD symptoms ${ }^{48}$.

We also report a significant association between genetic risk for lower thalamus volume and genetic risk for social disinhibition symptoms. Social disinhibition has been hypothesized to be an endophenotype that is relevant to OCD, TS and ADHD, reflecting deficits in top-down cognitive control across all of these conditions ${ }^{16}$. Given the involvement of the thalamus in many aspects of cognition, our findings are consistent with this hypothesis $^{39}$.

The association of genetic risk for greater putamen volume and genetic risk for symmetry symptoms suggests that the putamen may play a particularly important role in symmetry behaviours. CTSC circuitry certainly plays an important role in $\mathrm{TS}^{38}$ and it has been suggested that more complex tics-such as those involving symmetry behaviours - may be mediated by ventral striatal circuits ${ }^{49}$ that are also involved in $\mathrm{OCD}^{27,50}$. However, not all data are consistent; symmetry symptoms in TS may not share a significant degree of genetic overlap with $\mathrm{OCD}^{16}$ and findings from neuroimaging of symmetry symptoms in OCD have not emphasized striatal regions ${ }^{51,52}$.

The analyses here complement prior work on OCD, where we found significant concordance between $\mathrm{OCD}$ risk variants and variants that are related to greater putamen volume $\left(p=8.0 \times 10^{-4}\right)^{27}$. In addition, the CSTC has been implicated in both disorders ${ }^{38,53}$. Our previous study on OCD did, however, also identify significant concordance between greater nucleus accumbens volume and OCD and conditional FDR only revealed significant associations with OCD when conditioned on putamen, amygdala and thalamus volumes ${ }^{27}$. Differences between OCD and TS are consistent with genetic analyses which have emphasized that despite their relatedness, these conditions have different genetic architectures ${ }^{4,54}$. The findings here arguably support the proposal in the eleventh revision of the International Classification of Diseases (ICD-11) to classify TS as both a neurodevelopmental disorder and as an obsessive-compulsive related disorder (OCRD $)^{1,55}$.

The analysis here also complements previous work on ADHD; for example, the largest subcortical brain volume study of ADHD to date found lower putamen volumes as well as other alterations in this disorder ${ }^{56}$. Notably, although the putamen is also implicated in the TS symmetry cluster, the effect direction is opposite to that observed in $\mathrm{ADHD}^{56}$. Additional work on neuroimaging of children with TS may help clarify these differences.

The three variants associated with TS after conditioning on hippocampal (rs1922786) and ICV (rs2708146 and rs72853320) volumes have not previously been associated with a neuropsychiatric phenotype. Both SNPs, rs2708146 and rs1922786, are intronic variants located within the long intergenic non-protein coding RNA 1122 (LINC01122) gene on chromosome 2. The SNP, rs72853320, is an intergenic variant closest to the RNA U1 small nuclear 88 pseudogene (RNU1-88P) on chromosome 6. To date, little is known about the function of these genes, which are expressed in a broad range of tissue types, including the brain (https://www.ensembl.org/ index.html, accessed on 5 April 2018). Other variants within LINCO1122 have previously been associated with red blood cell count, mean corpuscular haemoglobin and mean corpuscular volume in a GWAS investigating blood cell phenotypes in the UK Biobank ${ }^{57}$. The study of blood cell phenotypes identified a total of 2706 variants associated with these phenotypes, located within or near genes that are involved in pathways that have been implicated in schizophrenia, autoimmune diseases and cardiovascular disorders ${ }^{57}$. Further, each of the three variants identified in the current study only accounted for $<1 \%$ of the variance observed in TS, as well as in each of the brain region volumes. Better powered studies are needed to address this issue.

Several limitations of this work should be emphasized. First, the LDSR analysis only lends trending, but not significant, support for the association between the thalamus and TS. LDSR was further underpowered to perform all the tests of brain volume with the TS symptom clusters. LDSR is ideally suited for datasets with more than 3000 samples, which was not the case for the TS symptom dimensions ${ }^{58}$. Estimating partitioned heritability typically requires datasets with at least 5000 samples $^{58}$. Approaches such as genome-wide complex trait analysis may be more applicable in future studies with these sample sizes, when individual-level data are 
available $^{58}$. LDSR requires larger datasets compared to SECA and cFDR as it determines genetic correlation in one direction of effect, whereas the latter techniques use the absolute size of effect and are able to distinguish whether the effect allele of a SNP confers risk for both traits or whether the effect directions are opposite for the traits $^{30,32,36}$. This also contributes to differences in the findings obtained across these techniques. Validation in an even larger cohort is necessary. Second, the TS GWAS that was used in this study had low power ${ }^{5}$ and may be susceptible to random variation. This is currently the largest dataset available for TS and as this dataset grows, replication studies will be possible. Third, the analysis could be biased if overlapping participants were present in the studies contributing to the consortia. We verified that the cohorts as whole did not overlap and individual overlap is, therefore, likely to be minimal. Fourth, the ENIGMA GWASs of brain volumes contain cohorts with healthy controls as well as patients diagnosed with neuropsychiatric disorders (including anxiety, Alzheimer's disease, ADHD, major depression, bipolar disorder, epilepsy and schizophrenia), which may bias the interpretation of our findings and how they relate to TS. However, a direct comparison of the GWAS summary statistics between the full ENIGMA results (including patients) and a subset of ENIGMA results (excluding patients) showed that they were very highly correlated $\left(r^{2}>0.99\right)$ for all brain traits ${ }^{26}$. This suggests that the pattern of effects in the brain volume GWAS is not likely driven by disease status. Fifth, approximately half of the TS cohort also has comorbid OCD. It is possible that stronger and more specific associations may be revealed in a cohort with TS only. Sixth, this study only investigated samples of European ancestry and the results are possibly only applicable to this population. Replication studies using other population groups are needed ${ }^{59}$. Seventh, the relationship between gene variants influencing brain volume and neuropsychiatric risk may be influenced by a range of confounders, including environmental factors such as stressors and medication effects, which may have effects on brain volume and disease risk independent of genetics. Discovering the pathway by which gene variants influencing brain volume also create risk for TS and its symptom clusters is therefore susceptible to influence by environmental factors, which might obscure genetic relationships. Future work on gene by environment interactions would be useful to clarify this. However, an endeavour to find the genetic overlap between brain volume and disorder risk using the largest datasets to date is still worthwhile, as it can potentially provide insights into the disorder.

In conclusion, this study implicated genetic overlap between genetic variants influencing the volumes of the thalamus, putamen and caudate in TS and its symptom dimensions. Further, this study identified three SNPs associated with TS and volumes of the hippocampus and ICV. Indeed, these data are the first to show an overlap between risk for genes for TS and for brain circuitry. The correlations with putamen and thalamus volumes are consistent with a broad range of previous neuroimaging work. Emerging collaborations and consortia, such as ENIGMA-TS, aim to continue to increase sample size, which will enhance statistical power in future iterations of this analysis. Additional work focusing on a range of other methodologies to assess genetic overlap may also be useful, following along the lines of recent work in schizophrenia ${ }^{60-62}$. Such studies have used partitioning-based heritability analysis ${ }^{63}$ and conjunction analysis ${ }^{64}$ to identify genetic variants associated with both schizophrenia risk and altered brain volumes. These approaches may also be useful in future work on TS and its symptom dimensions.

\section{Acknowledgements}

PGC-TS members: Harald Aschauer; Gil Atzmon; Cathy Barr; Csaba Barta; Robert Batterson; Fortu Benarroch; Chester Berlin; Gabriel Berrio; Julia Bohnenpoll; Lawrence Brown; Ruth Bruun; Randy Buckner; Cathy Budman; Julio Cardona Silgado; Danielle Cath; Keun-Ah Cheon; Sylvain Chouinard; Barbara Coffey; Giovanni Coppola; Nancy Cox; Sabrina Darrow; Lea Davis; Christel Depienne; Andrea Dietrich; Yves Dion; Valsamma Eapen; Lonneke Elzerman; Thomas Fernandez; Nelson Freimer; Odette Fründt; Blanca Garcia-Delgar; Donald Gilbert; Marco Grados; Erica Greenberg; Dorothy Grice; Varda Gross-Tsur; Julie Hagstrøm; Andreas Hartmann; Johannes Hebebrand; Tammy Hedderly; Gary Heiman; Luis Herrera; Isobel Heyman; Matthew Hirschtritt; Pieter Hoekstra; Hyun Ju Hong; Alden Huang; Chaim Huyser; Laura Ibanez-Gomez; Cornelia Illmann; Joseph Jankovic; Judith Kidd; Kenneth Kidd; Young Key Kim; YoungShin Kim; Robert King; Yun-Joo Koh; Anastasios Konstantinidis; Sodahm Kook; Samuel Kuperman; Roger Kurlan; James Leckman; Paul C. Lee; Bennett Leventhal; Thomas Lowe; Andrea Ludolph; Claudia Lührs da Silva; Gholson Lyon; Marcos Madruga-Garrido; Irene Malaty; Athanasios Maras; Carol A. Mathews; William McMahon; Sandra Mesa Restrepo; Pablo Mir; Astrid Morer; Kirsten Müller-Vahl; Alexander Münchau; Tara Murphy; Allan Naarden; Peter Nagy; Benjamin Neale; Markus Noethen; William Ochoa; Michael Okun; Lisa Osiecki; Peristera Paschou; David Pauls; Christopher Pittenger; Kerstin Plessen; Yehuda Pollak; Danielle Posthuma; Eliana Ramos; Victor Reus; Renata Rizzo; Mary Robertson; Veit Roessner; Josh Roffman; Guy Rouleau; Andres RuizLinares; Paul Sandor; Jeremiah Scharf; Monika Schlögelhofer; Eun-Young Shin; Harvey Singer; Jan Smit; Jordan Smoller; Dong-Ho Song; Jungeun Song; Mara Stamenkovic; Matthew State; Manfred Stuhrmann; Jae-Hoon Sul; Urszula Szymanska; Zsanett Tarnok; Jay Tischfield; Fotis Tsetsos; Jennifer Tübing; Ana Valencia Duarte; Frank Visscher; Sina Wanderer; Tomasz Wolanczyk; Martin Woods; Yulia Worbe; Dongmei Yu; Ivette Zelaya; Samuel Zinner. ENIGMA was supported in part by a Consortium grant (U54 EB020403 to PMT) from the NIH Institutes contributing to the Big Data to Knowledge (BD2K) Initiative, including the NIBIB and $\mathrm{NCl}$. The funders had no role in study design, data collection and analysis, decision to publish, or preparation of the manuscript. PGC-TS was supported by grants from the Judah Foundation, the Tourette Association of America, NIH Grants NS40024, NS016648, the American Recovery and Re-investment Act (ARRA) Grants NS040024-07S1, NS16648-29S1, NS040024-09S1, MH092289, MH092290, MH092291, MH092292, MH092293, MH092513, MH092516, MH092520, and the New Jersey Center for Tourette Syndrome and Associated Disorders (NJCTS). D.J.S. was supported by the SA Medical Research Council. N.G. was supported by the Claude Leon Foundation. M.S.M. was supported by the South African National Research Fund and the David and Elaine Potter Foundation.

\section{Author details}

${ }^{1}$ Human Genetics Research Unit, Division of Human Genetics, Department of Pathology, Institute of Infectious Disease and Molecular Medicine, Faculty of Health Sciences, University of Cape Town, Cape Town, South Africa. ${ }^{2}$ Imaging Genetics Center, Mark and Mary Stevens Neuroimaging \& Informatics Institute, Keck School of Medicine of the University of Southern California, Los Angeles, 
CA, USA. ${ }^{3}$ Department of Psychiatry and MRC Unit on Risk \& Resilience, University of Cape Town, Cape Town, South Africa. ${ }^{4}$ Groote Schuur Hospital and Neuroscience Institute, Cape Town, South Africa. ${ }^{5}$ Department of Psychiatry and the Behavioural Sciences, Zilkha Neurogenetic Institute, Keck School of Medicine, University of Southern California, Los Angeles, CA, USA. ${ }^{6}$ Department of Psychiatry, University of Stellenbosch, Stellenbosch, South Africa. ${ }^{7}$ Department of Biological Sciences, Purdue University, West Lafayette, IN, USA. ${ }^{8}$ Department of Psychiatry, Department of Anatomy \& Neurosciences, VU University Medical Center, Amsterdam Neuroscience, Amsterdam, Netherlands. ${ }^{9} \mathrm{Q} I M R$ Berghofer Medical Research Institute, Brisbane, Australia. ${ }^{10}$ Psychiatric and Neurodevelopmental Genetics Unit, Center for Genomic Medicine, Harvard Medical School, Boston, MA, USA. "'Department of Psychiatry, Massachusetts General Hospital, Boston, MA, USA. ${ }^{12}$ Department of Neurology, Massachusetts General Hospital, Boston, MA, USA. ${ }^{13}$ Department of Psychiatry, Genetics Institute, University of Florida, Gainesville, FL, USA

\section{Conflict of interest}

The authors declare that they have no conflict of interest.

\section{Publisher's note}

Springer Nature remains neutral with regard to jurisdictional claims in published maps and institutional affiliations.

Received: 12 July 2018 Revised: 13 February 2019 Accepted: 16 February 2019

Published online: 22 March 2019

\section{References}

1. Robertson, M. M. A personal 35 year perspective on Gilles de la Tourette syndrome: prevalence, phenomenology, comorbidities, and coexistent psychopathologies. Lancet Psychiatry 2, 68-87 (2015).

2. Hidalgo Vicario, M. H. \& Rodríguez Hernández, P. R. DSM-5. manual diagnóstico y estadístico de los trastornos mentales. últimas novedades. https://doi.org/ 10.1176/appi.books.9780890425596.744053 (2013).

3. Mataix-Cols, D. et al. Familial risks of tourette syndrome and chronic tic disorders a population-based cohort study. JAMA Psychiatry 72, 787-793 (2015)

4. Davis, L. K. et al. Partitioning the heritability of tourette syndrome and obsessive compulsive disorder reveals differences in genetic architecture. PLoS Genet. 9. https://doi.org/10.1371/journal.pgen.1003864 (2013).

5. Scharf, J. M. et al. Genome-wide association study of Tourette's syndrome. Mol. Psychiatry 18, 721-728 (2013).

6. Robertson, M. M. et al. Gilles de la Tourette syndrome. Nat. Rev. Dis. Prim. 3, 16097 (2017)

7. Grados, M. A. \& Mathews, C. A. Latent class analysis of Gilles de la Tourette syndrome using comorbidities: clinical and genetic implications. Biol. Psychiatry 64, 219-225 (2008).

8. Cheung, M. Y. C., Shahed, J. \& Jankovic, J. Malignant Tourette syndrome. Mov. Disord. 22, 1743-1750 (2007).

9. Jankovic, J., Gelineau-Kattner, R. \& Davidson, A. Tourette's syndrome in adults. Mov. Disord. 25, 2171-2175 (2010).

10. Patton, N. M. \& Holmes, H. T. Myxomatosis in domestic rabbits in Oregon. J. Am. Vet. Med. Assoc. 171, 560-562 (1977).

11. Paschou, P. et al. Genetic association signal near NTN4 in Tourette syndrome. Ann. Neurol. 76, 310-315 (2014).

12. Hirschtritt, M. E. et al. Social disinhibition is a heritable subphenotype of tics in Tourette syndrome. Neurology 87, 497-504 (2016).

13. De Haan, M. J., Delucchi, K. L., Mathews, C. M. \& Cath, D. C. Symptom dimensions and their heritabilities in Tourette's syndrome. Psychiatr. Genet. 25, 112-118 (2015).

14. Cavanna, A. E. et al. Dissecting the Gilles de la Tourette spectrum: a factor analytic study on 639 patients. J. Neurol. Neurosurg. Psychiatry 82, 1320-1323 (2011).

15. Robertson, M. M., Althoff, R. R., Hafez, A. \& Pauls, D. L. Principal components analysis of a large cohort with Tourette syndrome. Br. J. Psychiatry 193, 31-36 (2008).

16. Darrow, S. M. et al. Identification of two heritable cross-disorder endophenotypes for tourette syndrome. Am. J. Psychiatry 174, 387-396 (2017).
17. Felling, R. J. \& Singer, H. S. Neurobiology of Tourette syndrome: current status and need for further investigation. J. Neurosci. 31, 12387-12395 (2011).

18. Peterson, B. S. et al. Basal ganglia volumes in patients with Gilles de la Tourette syndrome. Arch. Gen. Psychiatry 60, 415-424 (2003).

19. Bloch, M. H. et al. Adulthood outcome of tic and obsessive-compulsive symptom severity in children with Tourette syndrome. Arch. Pediatr. Adolesc. Med. 160, 65-69 (2006).

20. Peterson, B. S. et al. Regional brain and ventricular volumes in Tourette syndrome. Arch. Gen. Psychiatry 58, 427-440 (2001).

21. Greene, D. J., Williams, A. C., Koller, J. M., Schlaggar, B. L. \& Black, K. J. Brain structure in pediatric Tourette syndrome. Mol. Psychiatry 22, 972-980 (2017).

22. Plessen, K. J. et al. Altered interhemispheric connectivity in individuals with Tourette's disorder. Am. J. Psychiatry 161, 2028-2037 (2004).

23. Plessen, K. J. et al. Reduced white matter connectivity in the corpus callosum of children with Tourette syndrome. J. Child Psychol. Psychiatry Allied Discip. 47, 1013-1022 (2006).

24. Miller, A. M. et al. Enlargement of thalamic nuclei in tourette syndrome. Arch. Gen. Psychiatry 67, 955-964 (2010).

25. Peterson, B. S. et al. Morphologic features of the amygdala and hippocampus in children and adults with Tourette syndrome. Arch. Gen. Psychiatry 64 1281-1291 (2007).

26. Hibar, D. P. et al. Common genetic variants influence human subcortical brain structures. Nature 520, 224-229 (2015).

27. Hibar, D. P. et al. Significant concordance of genetic variation that increases both the risk for obsessive-compulsive disorder and the volumes of the nucleus accumbens and putamen. Br. J. Psychiatry 213, 430-436 (2018).

28. Wakefield, J. C. DSM-5: An Overview of Changes and Controversies. https://doi. org/10.1007/s10615-013-0445-2 (2013).

29. Purcell, S. et al. PLINK: A tool set for whole-genome association and population-based linkage analyses. Am. J. Hum. Genet. 81, 559-575 (2007).

30. Nyholt, D. R. SECA: SNP effect concordance analysis using genome-wide association summary results. Bioinformatics 30, 2086-2088 (2014).

31. Ogino, $H$. et al. The synthetic rate of dipeptide catalyzed by organic solventstable protease from Pseudomonas aeruginosa PST-01 in the presence of water-soluble organic solvents. Biochem. Eng. J. 5, 219-223 (2000).

32. Andreassen, O. A. et al. Improved detection of common variants associated with schizophrenia and bipolar disorder using pleiotropy-informed conditional false discovery rate. PLoS. Genet. 9, e1003455 (2013).

33. Lawrence, R. J. Models of consumer purchasing behaviour. Source J. R. Stat. Soc. Ser. C. (Appl. Stat.) 15, 216-233 (1966).

34. Andreassen, O. A. et al. Improved detection of common variants associated with schizophrenia by leveraging pleiotropy with cardiovascular-disease risk factors. Am. J. Hum. Genet. 92, 197-209 (2013).

35. Bulik-Sullivan, B. et al. An atlas of genetic correlations across human diseases and traits. Nat. Genet. 47, 1236-1241 (2015).

36. Bulik-Sullivan, B. et al. LD score regression distinguishes confounding from polygenicity in genome-wide association studies. Nat. Genet. 47, 291-295 (2015).

37. Jeffries, K. J. et al. The functional neuroanatomy of Tourette's syndrome: an FDG PET study III: functional coupling of regional cerebral metabolic rates. Neuropsychopharmacology 27, 92-104 (2002).

38. Berardelli, A., Currà, A., Fabbrini, G., Gilio, F. \& Manfredi, M. Pathophysiology of tics and Tourette syndrome. J. Neurol. 250, 781-787 (2003).

39. Shipp, S. The brain circuitry of attention. Trends Cogn. Sci. 8, 223-230 (2004).

40. Porta, M. et al. Thalamic deep brain stimulation for treatment-refractory Tourette syndrome: two-year outcome. Neurology 73, 1375-1380 (2009).

41. Makki, M. I., Behen, M., Bhatt, A., Wilson, B. \& Chugani, H. T. Microstructural abnormalities of striatum and thalamus in children with tourette syndrome. Mov. Disord. 23, 2349-2356 (2008).

42. Marchand, W. R., Lee, J. N., Thatcher, J. W., Hsu, E. W., Rashkin, E. \& Suchy, Y. et al. Putamen coactivation during motor task execution. Neuroreport 19 957-960 (2008).

43. Ell, S. W., Marchant, N. L. \& Ivry, R. B. Focal putamen lesions impair learning in rule-based, but not information-integration categorization tasks. Neuropsychologia 44, 1737-1751 (2006).

44. Seger, C. A. The roles of the caudate nucleus in human classification learning. J. Neurosci. 25, 2941-2951 (2005)

45. Villablanca, J. R. Why do we have a caudate nucleus? Acta Neurobiol. Exp. (Wars.) 70, 95-105 (2010). 
46. Ludolph, A. G. et al. Grey-matter abnormalities in boys with Tourette syndrome: magnetic resonance imaging study using optimised voxel-based morphometry. Br. J. Psychiatry 188, 484-485 (2006).

47. Roessner, $\mathrm{V}$. et al. Increased putamen and callosal motor subregion in treatment-naive boys with Tourette syndrome indicates changes in the bihemispheric motor network. J. Child Psychol. Psychiatry Allied Discip. 52, 306-314 (2011).

48. Bloch, M. H., Leckman, J. F., Zhu, H. \& Peterson, B. S. Caudate volumes in childhood predict symptom severity in adults with Tourette syndrome. Neurology 65, 1253-1258 (2005).

49. de Vries, F. E. et al. Limbic and motor circuits involved in symmetry behavior in Tourette's syndrome. Cns. Spectr. 18, 34-42 (2013).

50. Radua, J., Van Den Heuvel, O. A., Surguladze, S. \& Mataix-Cols, D. Metaanalytical comparison of voxel-based morphometry studies in obsessivecompulsive disorder vs other anxiety disorders. Arch. Gen. Psychiatry 67 701-711 (2010).

51. Van Den Heuvel, O. A. et al. The major symptom dimensions of obsessivecompulsive disorder are mediated by partially distinct neural systems. Brain 132, 853-868 (2009).

52. Gilbert, A. R. et al. Neural correlates of symptom dimensions in pediatric obsessive-compulsive disorder: a functional magnetic resonance imaging study. J. Am. Acad. Child Adolesc. Psychiatry 48, 936-944 (2009).

53. Rauch, S. L. \& Britton, J. C. Developmental neuroimaging studies of OCD: the maturation of a field. J. Am. Acad. Child Adolesc. Psychiatry 49, 1186-1188 (2010).
54. Yu, D. et al. Cross-disorder genome-wide analyses suggest a complex genetic relationship between tourette's syndrome and OCD. Am. J. Psychiatry 172, 82-93 (2015).

55. Stein, D. J. et al. The classification of obsessive-compulsive and related disorders in the ICD-11. J. Affect Disord. 190, 663-674 (2016).

56. Hoogman, M. et al. Subcortical brain volume differences in participants with attention deficit hyperactivity disorder in children and adults: a cross-sectional mega-analysis. Lancet Psychiatry 4, 310-319 (2017).

57. Astle, W. J. et al. The allelic landscape of human blood cell trait variation and links to common complex disease. Cell 167, 1415-1429 (2016).

58. Ni, G. et al. Estimation of genetic correlation via linkage disequilibrium score regression and genomic restricted maximum likelihood. Am. J. Hum. Genet. 102, 1185-1194 (2018).

59. Rosenberg, N. A. et al. Genome-wide association studies in diverse populations. Nat. Rev. Genet. 11, 356 (2010).

60. Lee, P. H. et al. Partitioning heritability analysis reveals a shared genetic basis of brain anatomy and schizophrenia. Mol. Psychiatry 21, 1680-1689 (2016).

61. Franke, B. et al. Genetic influences on schizophrenia and subcortical brain volumes: Large-scale proof of concept. Nat. Neurosci. 19, 420-431 (2016).

62. Mufford, M. S. et al. Neuroimaging genomics in psychiatry-a translational approach. Genome Med. 9. https://doi.org/10.1186/s13073-017-0496-z (2017).

63. Yang, J., Lee, S. H., Goddard, M. E. \& Visscher, P. M. GCTA: a tool for genomewide complex trait analysis. Am. J. Hum. Genet. 88, 76-82 (2011).

64. Nichols, T., Brett, M., Andersson, J., Wager, T. \& Poline, J. B. Valid conjunction inference with the minimum statistic. Neuroimage 25, 653-660 (2005). 